\title{
AVALIAÇÃo dO USO de teCNOLOGIAS NO ATENDIMENTO DOMICILIAR DE CRIANÇAS E ADOLESCENTES NA CIDADE DE CURITIBA
}

\section{Evaluation of the use of technologies in children and teenagers home care in the city of Curitiba}

\begin{abstract}
Andrea Maria Cavalcante Alencar ${ }^{1}$, Elenize Losso² ${ }^{2}$ Talita Turatti do Carvalhal $^{3}$, Suzane
\end{abstract} Ketlyn Martello ${ }^{4}$

\begin{abstract}
1. Fisioterapeuta em Saúde da Família pelo Programa de Residência da SMS/ Feaes. Formada pelo Centro Universitário UNINOVAFAPI. ORCID: http://orcid.org/0000-0002-3581-1618

2. Fisioterapeuta no Programa Melhor em Casa de Curitiba. Mestre em Engenharia Biomédica pela UTFPR. ORCID: http://orcid.org/0000-0002-7643-4660

3. Fisioterapeuta no Programa Melhor em Casa formada na UNESP. Especialista em Fisioterapia Cardiorrespiratória. ORCID: http://orcid.org/0000-0003-0496-5152

4. Fisioterapeuta no Hospital de Dermatologia Sanitária do Paraná. Mestre em Tecnologia em Saúde, pela PUCPR. ORCID: http://orcid.org/0000-0003-4474-7045
\end{abstract}

CONTATO: Andrea Maria Cavalcante Alencar | Rua Luiz França, 1390 | Bairro Cajuru | Curitiba | Paraná | Brasil | CEP 82940-090 | E-mail: andrea.m.alencar@gmail.com

COMO CITAR Alencar AMC, Losso E, Carvalhal TT do, Martello SK. Avaliação do uso de tecnologias no atendimento domiciliar de crianças e adolescentes na cidade de Curitiba. R. Saúde Públ. 2018 Jul.;1(1):11-19.

(c) (i) COPYRIGHT Esta obra é disponibilizada nos termos da Licença Creative Commons - 4. 0 Internacional. É permitida a reprodução parcial ou total desta obra, desde que citada a fonte.

RESUMO Tendo em vista o crescente número de pacientes complexos atendidos em domicílio, o objetivo deste estudo foi avaliar a eficácia do uso de tecnologia em saúde, em pacientes de 0-17 anos, 11 meses e 29 dias, atendidos pelo serviço público de atenção domiciliar, Programa Melhor em Casa, da cidade de Curitiba - Paraná. Os seguintes dados foram coletados de planilhas e prontuários: uso e identificação de recurso tecnológico, tempo de permanência no programa, re-hospitalizações e dados clínicos e funcionais. A análise dos dados resultou em 40 participantes, predomínio do sexo masculino, idade média de $7( \pm 6)$ anos, e diagnósticos clínicos variados. 
A incidência de alteração de mecânica ventilatória foi de 50\% e o tempo de permanência no programa foi 82,5\% superior em pacientes com uso de tecnologia respiratória. A baixa incidência de re-hospitalizações pode indicar a efetividade do atendimento domiciliar possibilitando o uso consciente e seguro de tecnologia em domicílio.

RESUMO Saúde da Criança. Serviços de Assistência Domiciliar. Tecnologia Biomédica.

ABSTRACT Considering the growing number of complex patients treated at home, the objective of this study was to evaluate the efficacy of health technology in patients aged 0-17 years, 11 months and 29 days, attended by a public home care service, 'Programa Melhor em Casa' of the city of Curitiba - state of Paraná. The following data were collected from worksheets and medical reports: use and identification of technological resources, length of stay in the program, hospital readmissions, and clinical and functional data. Data analysis resulted in 40 participants, male predominance, mean age of $7( \pm 6)$ years, and varied clinical diagnoses. The incidence of altered ventilatory mechanics was $50 \%$ and the time spent in the program was $82.5 \%$ higher in patients with respiratory technology use. The low incidence of hospital readmissions may indicate the effectiveness of home care, allowing the conscious and safe use of homebased technology.

KEYWORDS: Child Health. Home Care Services. Biomedical Technology.

\section{INTRODUÇÃo}

$\mathbf{S}$

egundo a Portaria Consolidada $n^{\circ} 5$ de 28

de setembro de 2017, a Atenção Domiciliar (AD) é uma modalidade de atenção à saúde integrada à Rede de Atenção à Saúde (RAS), caracterizada por um conjunto de ações de prevenção e tratamento de doenças, reabilitação, paliação e promoção à saúde, prestadas em domicílio!.

A AD atualmente é dividida em modalidades específicas de atendimento. Segundo esta mesma Portaria, a prestação da assistência à saúde na modalidade AD1 é de responsabilidade, conjunta e articulada, das equipes de Atenção Primária, por meio de visitas regulares em domicílio, no mínimo, uma vez por mês.
No caso das modalidades AD2 e AD3. o Serviço de Atenção Domiciliar denominado Programa Melhor em Casa assegura atendimento a usuários que possuam problemas de saúde e dificuldade ou impossibilidade física de locomoção até uma Unidade de Saúde e que necessitem de maior frequência de cuidado ${ }^{1,23 .}$.

As diretrizes de políticas de saúde para o remanejamento de leitos hospitalares têm exigido a presença de programas de assistência domiciliar. sejam estes privados ou públicos. Assim, pacientes denominados clinicamente estáveis são enviados para seus lares e programas de desospitalização têm atuado na instrumentalização e assistência aos familiares, para que seja segura ao paciente $e^{2,13}$. A desospitalização ocorre em casos de pacientes que 
apresentam estabilidade clínica e funcional, cuidador responsável, ciência da seriedade do atendimento domiciliar, estrutura material e comunicação entre os componentes da Rede de Atenção à Saúde (Unidade de Saúde, Serviço de Atendimento Móvel de Urgência, Unidade de Pronto Atendimento e Hospital de origem), que devem ter ciência das necessidades do paciente visando providenciar insumos e atendimentos em caso de emergência ${ }^{2-4}$.

Além de critérios clínicos e de estrutura material, se faz necessário que a empresa responsável pelo fornecimento do material também forneça esterilização e troca periódica de materiais, além de profissionais responsáveis para manutenção e manuseio dos aparelhos, visando garantir um atendimento domiciliar sem riscos para o paciente p-7. $^{5}$

Há recursos que os fisioterapeutas podem encontrar em domicílios e que são de responsabilidade deste profissional ter o conhecimento prático e teórico que podem ser indispensáveis para a sobrevida. Citam-se: monitores e mensuradores de funções vitais, ventiladores pulmonares e seus componentes, geradores de pressão positiva, conhecidos como BIPAP e CPAP, cilindros e concentradores de oxigênio, reanimador manual, cânulas de traqueostomias e aspiradores de secreção ${ }^{8-12}$.

No Programa Melhor em Casa de Curitiba, os pacientes, incluindo crianças e adolescentes, são encaminhados de hospitais desta cidade e região metropolitana, avaliados pelo programa no hospital e admitidos em seu lar. Para esta admissão, é importante e necessária a participação da equipe de Atenção Primária responsável pelo paciente, uma vez que o Melhor em Casa possui caráter instrumentalizador e temporário, e as Unidades de Saúde são responsáveis permanentes pelo paciente.

Em resposta à solicitação feita à Comissão Nacional de Incorporação de Tecnologias no Sistema Único de Saúde (CONITEC), pela Secretaria de Atenção à Saúde do Ministério da Saúde, foi confirmada a incorporação da ventilação mecânica invasiva no Sistema Único de Saúde SUS, em outubro de $2017^{2}$.
Neste relatório, a CONITEC² avaliou e comparou os procedimentos domiciliares e hospitalares, observando: taxa de morte, necessidade de rehospitalização, liberação de leitos de Unidade de Terapia Intensiva UTI e custos. Viu-se que não houve diferença importante na taxa de morte entre os grupos, em relação à mesma doençabase. Sobre a necessidade de re-hospitalização, ela parece estar mais relacionada ao perfil/gravidade da doença do que ao tipo de ventilação mecânica utilizada (hospitalar/domiciliar). $\mathrm{O}$ uso da tecnologia domiciliar foi interpretado como uma alternativa para a liberação de leitos da UTI 2.6 .

As justificativas principais para o estudo decorrem de um crescente aumento do número de crianças e adolescentes em uso de tecnologia que estão recebendo atendimento domiciliar pelo Melhor em Casa Curitiba. Houve a necessidade de avaliar se o atendimento a essas crianças e adolescentes que fazem uso de tecnologia tem sido efetivo no município de Curitiba. A necessidade do remanejamento de leitos hospitalares e da implantação de programas de desospitalização são também justificativas para este estudo. Também deve lembrar-se da contribuição dos avanços tecnológicos na área da saúde na sobrevida, em situações em que os prognósticos não são favoráveis para cura, incluindo crianças e adolescentes $2,6,10$.

O objetivo primário do estudo foi avaliar a efetividade do uso da tecnologia em domicílio no cuidado ao paciente pediátrico. Os objetivos secundários foram: identificar o recurso tecnológico utilizado na assistência à criança e adolescente, em um Serviço de Atenção Domiciliar do município de Curitiba e verificar a incidência de reinternamentos hospitalares após a admissão do paciente no Programa Melhor em Casa Curitiba.

\section{METODOLOGIA}

Este estudo usou de metodologia retrospectiva, observacional e quantitativa. O levantamento bibliográfico foi realizado nas 
principais bases de pesquisa: Scielo, PubMed, Lilacs, Capes, Medline e Bireme, na janela de tempo de 2000 a 2017, nos idiomas Português, Inglês e Espanhol. As palavras-chave presentes nos Descritores de Saúde foram: Saúde da Criança, Serviços de Atenção Domiciliar, Tecnologia Biomédica. As Keywords foram: Child, Home Care Services, Biomedical Technologies. Também foram empregados os operadores booleanos "e" e "ou" e inclusos os seguintes estudos: revisão bibliográfica, descritivo, observacional, qualitativos, quantitativos e foram excluídos estudos experimentais realizados tanto em animais quanto em seres humanos.

Após pesquisa bibliográfica, os sujeitos selecionados para coleta de dados foram: pacientes pediátricos e adolescentes, 0 a 17 anos, 11 meses e 29 dias, de ambos os sexos, com patologias multivariadas, internados de 2012 a 2017, no Melhor em Casa Curitiba, provenientes de hospitais desta cidade e região metropolitana.

Os dados coletados para este estudo foram: sexo, idade, diagnóstico clínico e cinético-funcional, número de atendimentos realizados pelas equipes multiprofissionais, tempo de internamento domiciliar, número de reinternamentos, intervalo entre internamentos, identificação do recurso tecnológico utilizado, identificação do tipo de via aérea, do uso de oxigênio associado e sua titulação.

Quanto ao tipo de via aérea, com ou sem via aérea artificial, que nestes pacientes eram cânulas traqueais: tipo de cânula traqueal, de material metálico ou plástico, numeração e cuff. Do recurso tecnológico identificaram-se órteses, cadeira de rodas, muletas, bengalas, concentradores, torpedos de oxigenoterapia, oxímetros, aspiradores, cateteres, máscara e equipamentos de ventilação mecânica. Quanto aos diagnósticos funcionais: hemiplegia, hemiparesia, paraplegia, tetraplegia ou quadriplegia, paraparesia, tetraparesia ou quadriparesia, déficit de mobilidade, déficit de coordenação, déficit de força muscular e déficit de equilíbrio, atraso no desenvolvimento neuropsicomotor (DNPM), hipersecretividade pulmonar e alteração da mecânica ventilatória.
O projeto foi aprovado pelo Comitê de Ética em Pesquisa (CEP) com CAAE 70741217400000101, em 21 de junho de 2017, e a coleta teve início após esta aprovação, utilizando planilhas computadorizadas dos atendimentos fisioterapêuticos e de prontuários físicos e eletrônicos.

\section{ANÁLISE ESTATÍSTICA}

A análise estatística dos dados foi realizada por meio do software IBM SPSS Statistics (v.21.0). A normalidade da distribuição das variáveis quantitativas contínuas foi testada por meio do teste de Kolmogorov-Smirnov. O teste não paramétrico de Mann-Whitney foi aplicado para comparar o tempo de permanência no Programa Melhor em Casa, $n^{\circ}$ de atendimentos realizados pelo SAD e número de reinternações, com relação ao uso de recursos tecnológicos (sim ou não) e ao sexo. Em todas as análises, um nível de significância de $5 \%(\alpha=0,05)$ foi assumido. Os dados foram apresentados por meio de média, desvio padrão, frequências absolutas e percentuais relativos.

\section{RESULTADOS}

A amostra foi composta por 40 participantes, com idade entre 0 e 17 anos,11 meses e 29 dias, inseridos no Melhor em Casa Curitiba, cujas características estão apresentadas na Tabela 1 por meio de média, desvio padrão (DP), frequências absolutas e percentuais relativos.

Dos 40 prontuários analisados, 24 eram do sexo masculino e 16 do sexo feminino, com idade média geral de $7( \pm 6)$ anos. O tempo médio de permanência no SAD foi de 199,3 ( \pm 306,3) dias, com um número médio de atendimentos de 72,4 ( \pm 98,6) e uma frequência de internação média de 1,1 $( \pm$ 1,8). Com relação à oxigenoterapia, esta foi utilizada por $62,5 \%$ dos participantes. $62,5 \%$ fizeram uso de ventilação mecânica, das quais 68,0\% era invasiva. A traqueostomia foi necessária para 52,5\% dos sujeitos, das quais, 85,7\% eram plásticas. Quanto à numeração da traqueostomia e a presença de cuff, os dados não estavam presentes na maioria dos prontuários e por isso não foram coletados. 
Tabela 1. Características da amostra.

\begin{tabular}{|c|c|c|}
\hline \multicolumn{2}{|l|}{ VARIÁVEIS } & $\begin{array}{c}\text { MÉDIA } \pm \text { DP } / \\
\text { FREQ (\%) }\end{array}$ \\
\hline \multicolumn{2}{|l|}{ Idade (anos) } & $7 \pm 6$ \\
\hline \multirow[t]{2}{*}{ Sexo } & Fem & $16(40,0)$ \\
\hline & Masc & $24(60,0)$ \\
\hline \multirow[t]{2}{*}{ Uso de traqueostomia } & Sim & $21(52,5)$ \\
\hline & Não & $19(47,5)$ \\
\hline \multirow[t]{2}{*}{ Tipo de traqueostomia } & Plástica & $18(85,7)$ \\
\hline & Metálica & $3(14,7)$ \\
\hline \multirow[t]{2}{*}{ Uso de oxigênio } & Sim & $25(62,5)$ \\
\hline & Não & $15(37,5)$ \\
\hline \multirow[t]{2}{*}{ Uso de VM } & Sim & $25(62,5)$ \\
\hline & Não & $15(37,5)$ \\
\hline \multirow[t]{2}{*}{ Tipo de VM } & Invasiva & $17(68,0)$ \\
\hline & Não Invasiva & $8(32,0)$ \\
\hline \multicolumn{2}{|l|}{$\mathrm{N}^{\circ}$ de reinternações } & $1,1 \pm 1,8$ \\
\hline \multicolumn{2}{|c|}{ Tempo de permanência no SAD (dias) } & $199,3 \pm 306,3$ \\
\hline \multicolumn{2}{|c|}{$\mathrm{N}^{\circ}$ de atendimentos realizados pelo SAD } & $72,4 \pm 98,6$ \\
\hline
\end{tabular}

SAD: Serviço de Atenção Domiciliar - Programa Melhor em Casa.

Fonte: Elaborada pelos autores (2018)

Na Tabela 2 estão apresentadas as comparações entre o tempo de permanência no programa, o número de atendimentos e a frequência de reinternações entre quem fez ou não o uso de algum recurso tecnológico.

Diferenças significantes estatisticamente foram encontradas para o tempo de permanência no Programa Melhor em Casa e o $\mathrm{n}^{\circ}$ de atendimentos realizados. Os indivíduos que não fizeram uso de recurso tecnológico tiveram um tempo de permanência média no programa de 51,9 dias, nos quais foram realizados em média 16,4 atendimentos pelo SAD. Já aqueles que precisaram de algum tipo de recurso tecnológico tiveram um tempo de permanência $80 \%$ superior, com uma média de 255,2 dias, nos quais foram realizados 93,7 atendimentos, ou seja, 82,5\% a mais. Já em relação à frequência de reinternações hospitalares, não foram observadas diferenças significativas. Os recursos tecnológicos estão apresentados na Figura 1 , com as respectivas frequências de utilização.

Conforme pode ser observado na Figura 1, o recurso isolado mais utilizado foi a oxigenoterapia, relatado em 25\% dos prontuários. Ao somar os casos em que a oxigenoterapia é usada em associação a outros recursos, esse percentual chega a 55\%. A VMNI isolada foi utilizada por apenas 5\% dos participantes, porém, em associação a outro recurso, foi relatada em 30\% dos prontuários levantados. A associação da VMNI à oxigenoterapia,

Tabela 2. Comparação do tempo de permanência no Programa Melhor em Casa, $n^{\circ}$ de atendimentos e reinternações conforme o uso ou não de recurso tecnológico.

\begin{tabular}{l|cc|c}
\hline \multirow{2}{*}{ VARIÁVEIS } & \multicolumn{2}{|c|}{ USO DE RECURSO TECNOLÓGICO } & \multicolumn{1}{c}{$\boldsymbol{p}$} \\
& SIM & NÃO & \\
\hline Tempo de permanência no SAD (dias) & $255,2 \pm 344,0$ & $255,2 \pm 344,0$ & $0,001^{*}$ \\
$N^{0}$ de atendimentos realizados pelo SAD & $93,7 \pm 108,1$ & $93,7 \pm 108,1$ & $0,001^{*}$ \\
N $^{0}$ de reinternações & $1,4 \pm 2,0$ & $1,4 \pm 2,0$ & 0,103 \\
\hline
\end{tabular}

SAD: Serviço de Atenção Domiciliar - Programa Melhor em Casa Curitiba; *: significância estatística; Teste de Mann-Whitney ( $\alpha=0,05$ ). 
Figura 1. Frequência de utilização dos recursos tecnológicos.

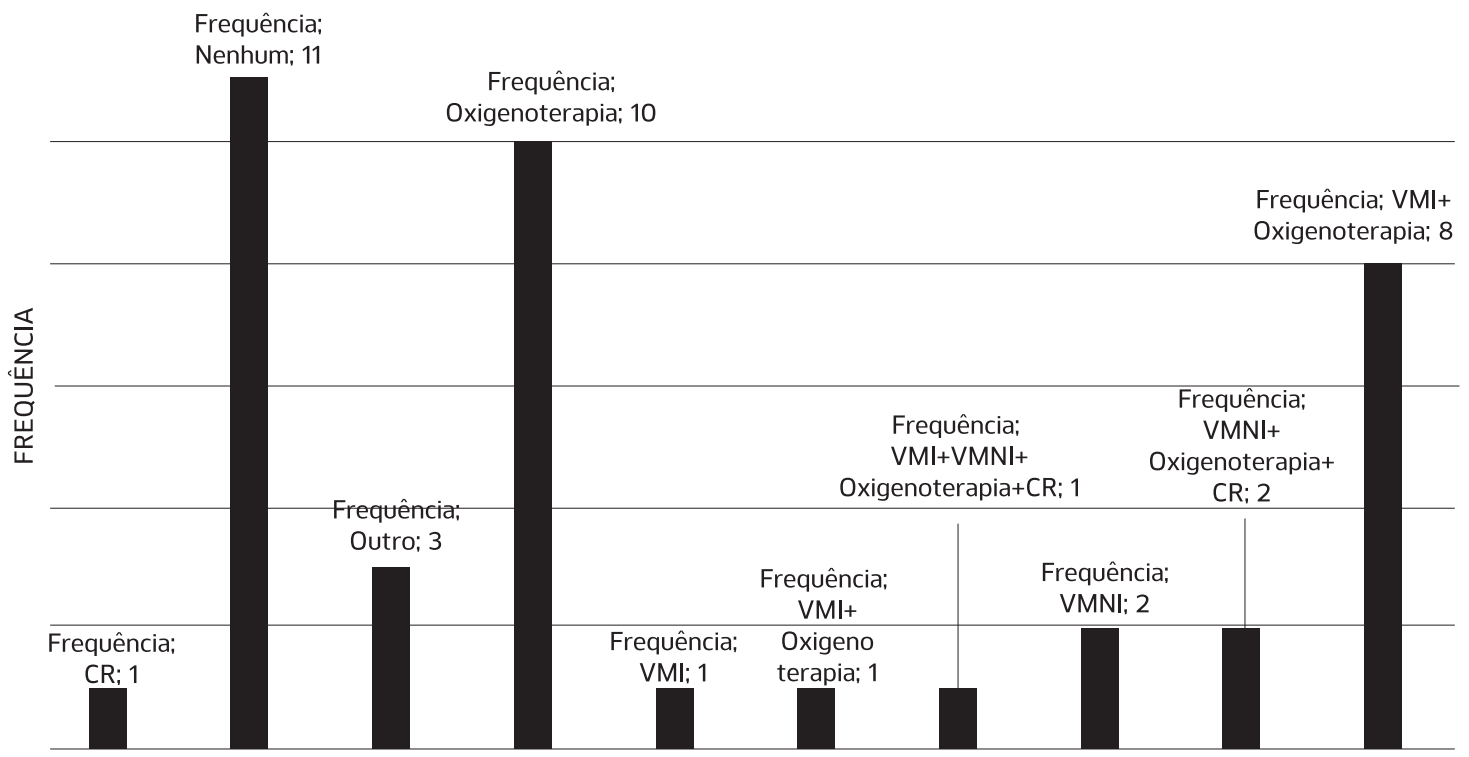

CR: cadeira de rodas; VMI: ventilação mecânica invasiva; VMNI: ventilação mecânica não invasiva. Fonte: Elaborada pelos autores (2018)

especificamente, foi aplicada em 20\% dos participantes. A categoria "Outro" refere-se ao uso de colar cervical, órtese de posicionamento para membro inferior e tipoia canadense.

Procurou-se investigar ainda se havia diferença no tempo de permanência no Programa Melhor em Casa, no $\mathrm{n}^{\circ}$ de atendimentos e de reinternações, de acordo com o sexo. Os resultados estão apresentados na Tabela 3.
Nenhuma das variáveis testadas apresentou diferença significante estatisticamente entre os sexos. Apesar do tempo de permanência no programa ter sido $31 \%$ superior entre os participantes do sexo masculino, vale a pena ressaltar os altos valores de desvio padrão em ambos os sexos. Além disso, os participantes do sexo masculino corresponderam a 60\% da amostra.

Tabela 3. Comparação do tempo de permanência no Programa Melhor em Casa, $n^{\circ}$ de atendimentos e reinternações de acordo com o sexo.

\begin{tabular}{|c|c|c|c|}
\hline VARIÁVEIS & FEMININO & MASCULINO & $p$ \\
\hline Tempo de permanência no SAD (dias) & $167,7 \pm 274,0$ & $220,3 \pm 330,1$ & 0,174 \\
\hline $\mathrm{N}^{\circ}$ de atendimentos realizados pelo SAD & $65,6 \pm 114,2$ & $77,0 \pm 89,0$ & 0,304 \\
\hline $\mathrm{N}^{\circ}$ de reinternações & $0,94 \pm 2,0$ & $1,1 \pm 1,8$ & 0,576 \\
\hline
\end{tabular}

SAD: Serviço de Atenção Domiciliar - Programa Melhor em Casa Curitiba; Teste de Mann-Whitney $(\alpha=0,05)$. Fonte: Elaborada pelos autores (2018) 
Figura 1. Frequência de utilização dos recursos tecnológicos.

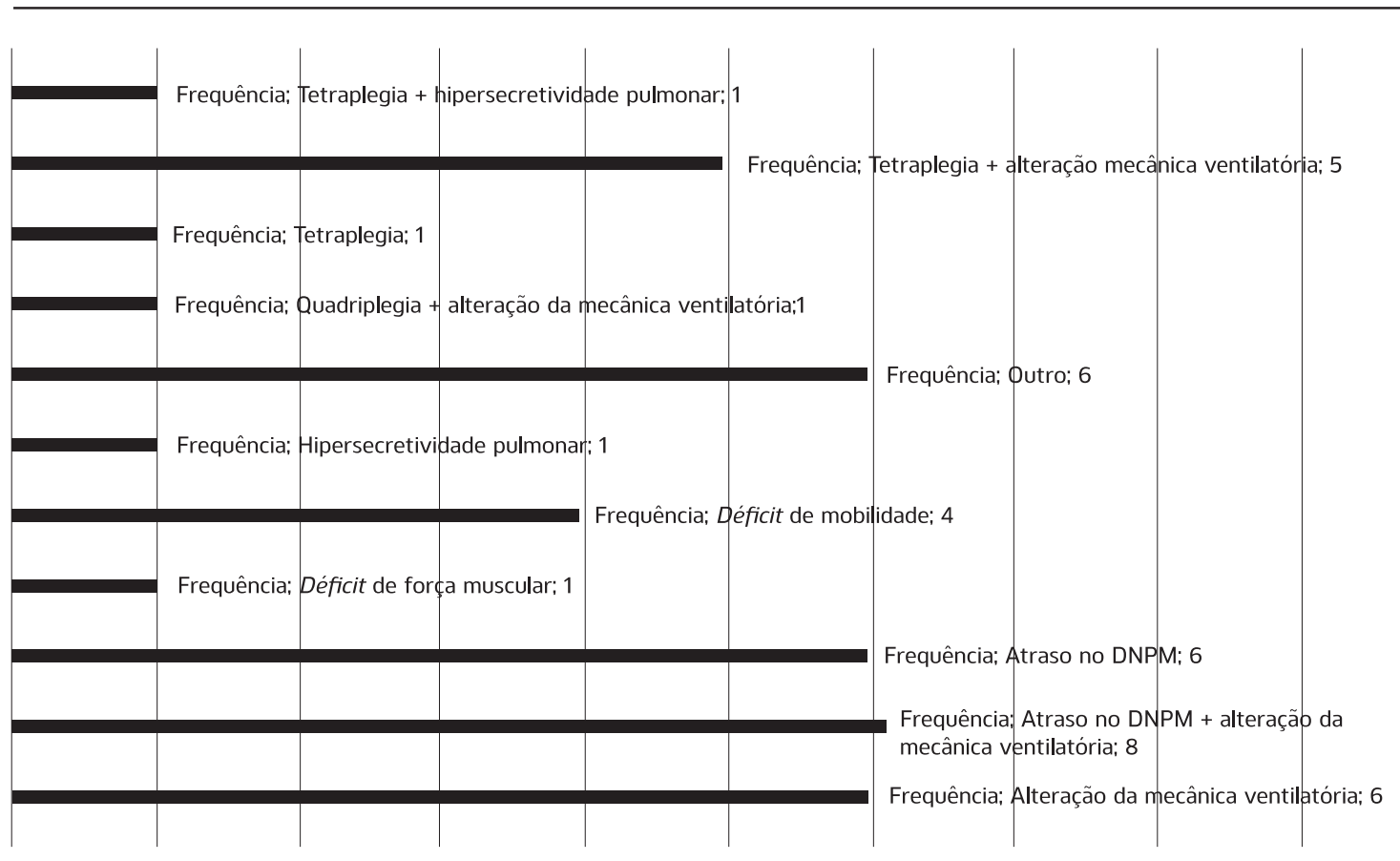

CR: cadeira de rodas; VMI: ventilação mecânica invasiva: VMNI: ventilação mecânica não invasiva

Fonte: Elaborada pelos autores (2018)

Os diagnósticos clínicos dos participantes foram demasiadamente variáveis, não sendo possivel a apresentação de suas frequências e percentuais relativos. A Figura 2 apresenta os diagnósticos funcionais relatados nos prontuários com as respectivas frequências.

Assim como na análise dos recursos tecnológicos, os diagnósticos funcionais foram apresentados tanto de forma isolada quanto quando em associação a outros diagnósticos, conforme relatado em prontuário. O atraso no DNPM em associação à alteração da mecânica ventilatória foi o mais frequente (20\%), seguido pela alteração da mecânica ventilatória isolada e "outro" diagnóstico, ambos representando 15\% da amostra. Ao agrupar todas as categorias que apresentam alteração na mecânica ventilatória, esse percentual chega a 50\% dos participantes.

\section{DISCUSSÃO}

O presente estudo apresentou uma avaliação do uso de tecnologia no domicílio realizado pelo Programa Melhor em Casa, ao paciente pediátrico e adolescente, com quadro clínico complexo e com necessidade de tecnologia especializada.

Dos 40 prontuários levantados, 24 participantes eram do sexo masculino e 16 do sexo feminino, com idade média geral de $7( \pm 6)$ anos. No estudo de Resener, Martinez, Reiter e Nicolai13, os pacientes apresentaram idades entre 1 e 21 anos, sendo 11 do sexo masculino e 15 do feminino.

Com relação à oxigenoterapia, no presente estudo, foi utilizada por $62,5 \%$ dos participantes e que fizeram uso de ventilação mecânica, das quais $68,0 \%$ era invasiva. A traqueostomia foi necessária para 52,5\% dos sujeitos, das quais, 85,7\% eram plásticas, pois dependiam de uso contínuo de ventilação. Resener et al. ${ }^{13}$ apontou 
12 crianças (46,2\%) ventiladas através de técnicas não invasivas e 19 (73,1\%) necessitavam apenas de suporte ventilatório intermitente.

No estudo de Garcia, Mezzacappa e Pessoto ${ }^{14}$. a ventilação mecânica invasiva foi utilizada por 68.0\% dos participantes e no estudo de Hanashiro ${ }^{10}$. 08 crianças em ventilação invasiva proporcionariam uma otimização de 12 leitos hospitalares por mês.

No estudo de Garcia et al. ${ }^{14}$, além de oxigênio inalatório, 127 casos (83\%) utilizaram ventilação mecânica invasiva. A média \pm DP para esta variável foi de 20,2 2 19,6 dias, variando de 1 a 86 dias. Quanto ao uso de pressão positiva contínua nas vias aéreas (CPAP), 88 casos (58\%) utilizaram esta modalidade, com duração média de 5,5 \$5,3 dias.

Quanto ao tempo de permanência no programa, foi $80 \%$ superior diante de paciente complexo e com uso de tecnologia em domicílio, exigindo um maior número de atendimentos pela equipe.

Neste estudo, houve diversas patologias crônicas, caracterizadas como alta complexidade, exigindo cuidados especiais para a família e equipe de assistência domiciliar. Os dados da incidência de patologias foram muito variados, não permitindo uma identificação de frequências. Nas literaturas de Garcia et al. ${ }^{14}$ e Munhoz et al. ${ }^{15}$ houve um predomínio de patologias de comprometimento respiratório, fibrose cística, displasia broncopulmonar e bronquiolite obliterante. Em pesquisa realizada pelo Hospital Alemão Oswaldo Cruz, as principais patologias encontradas foram de causas pulmonares e neurológicas².

Assim como na análise dos recursos tecnológicos, os diagnósticos funcionais foram apresentados tanto de forma isolada quanto quando em associação a outros diagnósticos, conforme relatado em prontuário. O atraso no DNPM em associação à alteração da mecânica ventilatória foi o mais frequente (20\%), seguido pela alteração da mecânica ventilatória isolada e "outro" diagnóstico, ambos representando 15\% da amostra. Resener et al. $^{13}$ relataram quanto às patologias que levaram à Insuficiência respiratória crônica (IRC), 15 crianças (57,7\%) apresentavam patologias neuromusculares com alteração da função dos músculos respiratórios, 8 pacientes (30,8\%) tinham como diagnóstico problemas ventilatórios de causa central e 3 crianças (11,5\%), doenças pulmonares obstrutivas.

O tempo de permanência no programa foi 80\% superior diante de paciente complexo e com uso de tecnologia em domicílio, exigindo-se maior número de atendimentos pela equipe.

Sobre o tempo de permanência no Melhor em Casa (Tabela 2): Hanashiro observou que a ventilação mecânica invasiva de oito pacientes disponibilizou 4.022 leitos-dia no hospital em 4 anos (uma média de 12 novos pacientes por mês na UTI), e a taxa de sobrevida dos pacientes em casa não foi significativamente diferente daquela verificada nos pacientes hospitalizados ${ }^{2,10}$.

Resener et al.13 mostraram a distribuição de acordo com o tempo decorrido desde a indicação da ventilação domiciliar, onde 20 das 26 crianças (76,9\%) estavam sendo ventiladas há menos de 5 anos. Quatro crianças (15,5\%) estavam em ventilação domiciliar por período de tempo entre 7 e 9 anos, praticamente desde o início do programa.

Dos recursos encontrados no estudo realizado no Melhor em Casa Curitiba, conforme a Tabela 1, predominou a tecnologia de mecânica respiratória invasiva. No estudo de Resener et al. ${ }^{13}$, de 26 crianças, 12 eram ventiladas de modo contínuo e não invasivo e 19 necessitavam apenas de ventilação intermitente, diferente deste estudo em que apenas 5\% fizeram uso de ventilação não invasiva .

Conforme a Conitec ${ }^{2}$, os equipamentos são classificados em três níveis ou especificações: 1-representados por equipamentos mais simples do tipo geradores de pressão positiva; 2-equipamentos do tipo bipap ou bilevel com ou sem baterias e 3para suporte de vida; dotados de bateria interna com duração de 04 a 11 horas.

Quanto ao uso de oxigênio suplementar, a principal forma de fornecimento de oxigênio no município de Curitiba ocorre por meio de concentradores, diferente do relatório do Conitec, onde prevalece o uso de cilindros ${ }^{2}$. 
Em relação aos reinternamentos ou rehospitalizações, não foram observadas diferenças significativas entre os indivíduos que não tiveram nenhum uso de recurso tecnológico e os que utilizaram algum tipo de recurso tecnológico. Porém, de forma isolada, a taxa de reinternamento apresentou baixo percentual nos pacientes assistidos em domicílio, o que mostra a efetividade do serviço prestado e da corresponsabilização do cuidador e de todos os outros componentes da RAS.

\section{CONCLUSÃO}

Após análise detalhada foi possível identificar os recursos tecnológicos mais utilizados e a incidência de reinternamentos hospitalares durante a assistência domiciliar ao paciente pediátrico no município de Curitiba. Em suma, pôde-se verificar que a complexidade dos diagnósticos apresentados pelos pacientes estava diretamente relacionada com a necessidade do uso de tecnologia mecânica respiratória invasiva. Além disso, a baixa incidência de reinternamentos hospitalares comprova a efetividade do atendimento domiciliar possibilitando o uso consciente de tecnologia em domicílio.

A assistência em saúde associada ao uso de tecnologias realizada em domicílio é algo complexo e relativamente novo no país e por isso se faz necessária a constante formalização de fluxos e rotinas entre os componentes da RAS para que esse tipo de assistência seja ágil e segura. A confecção de pesquisas científicas a respeito do assunto pode gerar constatações pertinentes que reforcem a $A D$, de qualidade, como meio de garantir a desospitalização de pacientes com ou sem uso de tecnologias; e consequentemente a liberação de leitos hospitalares

\section{REFERÊNCIAS}

1. Brasil. Ministério da Saúde. Portaria de Consolidação $n^{\circ} 5$, de 28 de setembro de 2017. Consolidação das normas sobre as ações e os serviços de saúde do Sistema Único de Saúde. Diário Oficial da União, Brasília (DF), 03 out; 2017
2. Brasil. Ministério da Saúde. Secretaria de Ciência, Tecnologia e Insumos Estratégicos. Comissão Nacional de Incorporação de Tecnologia no SUS (CONITEC). Brasília: Ministério da Saúde; 2017.

3. Lyra Filho FJ, Hirschheimer MR. Atenção domiciliar a crianças e adolescentes. Recomendações. Atualizações de Condutas em Pediatria. São Paulo: Sociedade de Pediatria de São Paulo; 2010.

4. Salgado EQMA, Luis LAF, Leite NSL. A família da criança dependente de tecnologia. In: Assistência Domiciliar Pediátricatrabalho interdisciplinar, conceitos e desafios em dependências tecnológicas. São Paulo: Atheneu; 2013.

5. Lima TC, Vargas MAO. Cuidado domiciliar intensivo: uma possivel realidade do Sistema Único de Saúde? Rev Bras Enferm 2004:57(6):658-61.

6. Pessanha FB, Freitas LAV, Rodrigues M, Mendes RAG Abordagem fisioterapêutica global buscando a integridade das crianças dependentes de tecnologias. In: Cruz Filho AD, Vachod L. Assistência Domiciliar Pediátrica-trabalho interdisciplinar conceitos e desafios em dependências tecnológicas. São Paulo: Atheneu; 2013

7. Carbonero FC, Zago GM, Campos D. Tecnologia assistiva na Distrofia Muscular de Duchenne:aplicabilidade e benefícios. Rev Neurocienc.2012;20(1):109-16.

8. Drucker LP. Home technological support network for technolog dependent children discharged from a state-run hospital. Ciênc saúde coletiva. 2007:12(5):1285- 94.

9. Adde FV, Alvarez AE, Barbisan BN, Guimarães BR Recommendations for long-term home oxygen therapy in children and adolescents. J Pediatr. 2013; 89(1):6-17.

10. Hanashiro M, Franco AOC, Ferraro AA, Troster EJ. Alternativas de tratamento para pacientes pediátricos em ventilação mecânica crônica. J Pediatr. 2011;87(2):145-9.

11. França EET, Ferrari FR, Fernandes PV, Cavalcanti R; Duarte A. Aquim EE et al. Força tarefa sobre a fisioterapia em pacientes críticos adultos: Diretrizes da Associação Brasileira de Fisioterapia Respiratória e Terapia Intensiva (ASSOBRAFIR) e Associação de Medicina Intensiva Brasileira. São Paulo: Associação de Medicina Intensiva Brasileira; 2012.

12. Barbas CS, Ísola AM, Farias AM, Cavalcanti AB, Gama AM Duarte AC, et al. Recomendações brasileiras de ventilação mecânica 2013. J Bras Pneumol. 2014:40(5):458-86.

13. Resener TD, Martinez FE, Reiter K, Nicolai T. Assistência ventilatória domiciliar em crianças: descrição de um programa. J. Pediatr. 2001;77(2): 84-88

14. Garcia EAL, Mezzacappa MA, Pessoto MA. Programa de oxigenoterapia domiciliar para crianças egressas de uma unidade neonatal: relato da experiência de dez anos. Rev Paul Pediatr.2010:28(3):276-82

15. Munhoz AS, Adde FV, Nakaie CMA, Doria Filho U, Silva Filho LVRF, Rodrigues JC. Oxigenoterapia domiciliar prolongada em crianças e adolescentes: uma análise do uso clínico e de custos de um programa assistencial. J. Pediatr. 2011;87(1):13-8. 